\title{
Low-cost generic drug programs in the US: implications for payers and researchers
}

\author{
Joshua D Brown, PharmD, MS
}

Low-cost generic drug programs (LCGPs) provide affordable generics in the US. However, LCGPs have implications for managed care organizations and researchers relying on claims data.

Keywords: Generic medications, low-cost generic drug programs, quality assessment, United States healthcare system

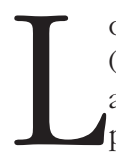

ow-cost generic drug programs (LCGPs) in the US increase the affordability and accessibility of prescription medication [1]. LCGPs are unique to the US market as a loss-leader pricing strategy, i.e. retailers accept a loss on these cheap medications to bring in customers, used by eight of the top 10 pharmacy chains, e.g. Wal-Mart, Walgreens, Rite Aid; providing many of the most commonly used generic medications at copayments of US\$4-5 for 30-day supplies or US\$10-12 for 90-day supplies [1-3]. These prices are much lower than the copayment for the medications; thus, patients using these programmes acquire the medications without the insurance company's knowledge.

Our group recently assessed the prevalence and patient characteristics associated with LCGP use in the US among those who are privately [4] and publicly (Medicare) [5] insured as well as in uninsured [6] and paediatric [7] populations. Within each group, we analysed which medications are most commonly purchased through LCGPs, the prevalence of LCGP use at the individual level, and the predictors of LCGP use in a nationally representative sample. Most clear from these studies is that there is a high prevalence of use beyond what was previously known with $36.4 \%$ of privately insured adults, $37.9 \%$ of older Medicare beneficiaries, $39.9 \%$ of those who are uninsured, and $23.7 \%$ of children and adolescents using LCGP medications.

The high utilization of these programmes has sweeping implications, especially in the insured adult and insured elderly populations. By using these programmes, no information is submitted through an individual's insurance benefit; thus, medication use data can be missing from administrative claims data. In the US, claims data are widely used as a primary source for health plans to assess their quality of care and for quality measurement, for pharmacovigilance and safety surveillance, as well as for research purposes for pharmacoepidemiologic [8].

Quality measurement is mandated by the government for publicly funded insurance programmes offered through managed care organizations (MCOs) and is based on a set standard of measures - including some measures of pharmaceutical utilization [9, 10]. Given the multiple levels of care in the healthcare system, these measures have also trickled down to affect provider prescribing quality, as well [11]. LCGPs can be implicated when these plans and providers attempt to measure their quality of care for, as an example, diabetic or postmyocardial infarction patients. The rates at which metformin (16-30\%), angiotensin converting enzyme inhibitors (ACE inhibitors; $17-30 \%)$; sulfonylureas (14-25\%), and beta-blockers (11-23\%) are filled through these programmes are tremendous. Thus, each medication filled through LCGP programs goes unobserved in claims data. This will lead to an underestimation of overall quality and a lower quality score, which becomes important given that these scores have been linked to plan enrolment and can impact quality-based reimbursement packages in a 'pay-for-performance' healthcare environment [12, 13].

MCOs are beginning to investigate LCGPs as a source of prescription drugs and are desperately searching for ways to curb their use so they can limit the loss of information for quality measurement. However, limiting access to prescription medications through LCGPs cannot be an effective solution given that increasing medication costs could be a barrier to treatment or patient adherence to treatment. Rather, MCOs should work with pharmacy providers to ensure that claims are submitted for these medications, which could be incentivized by including these cheaper generic drug prices as covered costs under the prescription benefit. Otherwise, a system wide change is likely needed to account for the use of LCGP medications, which would need to be part of a Centers for Medicare and Medicaid Services (CMS) mandate to enact a solution to this important issue.

Beyond affecting the bottom-line of MCOs, the implications of LCGP use also extend to those using claims data for signal detection of harmful medications and research. The well known US Food and Drug Administration's (FDA) Sentinel Initiative is a conglomeration of several claims databases used as a means of medication safety surveillance [14]. Similarly, researchers use claims data for pharmaco-epidemiologic research investigating the harms or benefits of medications. For these types of applications, exclusion of medication exposures introduces exposure misclassification bias when use of the medication of interest is incorrectly assigned [15]. This type of bias nearly always biases an effect measure to

Author: Joshua D Brown, PharmD, MS, Institute for Pharmaceutical Outcomes \& Policy, University of Kentucky College of Pharmacy, Lexington, KY 40535, USA

Submitted: 7 March 2016; Revised: 5 April 2016; Accepted: 5 April 2016; Published online first: 18 April 2016 
the null hypothesis, i.e. it underestimates the true association between the outcome and the medication. The impact of this bias for a harmful effect would then be to increase the chances of accepting a false null hypothesis that the medication is not harmful when it truly is, or for a protective effect it would find that the medication was not protective when it in fact may be. The size of this bias is a function of the proportion of the sample misclassified and the true effect size. The implications of this bias can be tremendous for medication classes used for prevention of negative health outcomes or medications that are associated with serious adverse events. For researchers, awareness of the issue is paramount to conducting a robust study and the astute researcher should use multiple sensitivity analyses or proxy measures to validate and strengthen their findings.

Much more research is needed to assess LCGPs including the overall impact on the quality measurement system, cost savings to patient and MCOs, and examples where reassessment of research findings may be necessary. One thing can be certain, LCGPs are likely to remain given the high consumer demand for cheaper access to medications.

\section{Competing interests: None.}

Provenance and peer review: Not commissioned, externally peer reviewed.

\section{References}

1. Choudhry NK, Shrank WH. Four-dollar genericsincreased accessibility, impaired quality assurance. N Engl J Med. 2010;363(20):1885-7.

2. Czechowski JL, Tjia J, Triller DM. Deeply discounted medications: implications of generic prescription drug wars. J Am Pharm Assoc (2003) 2010;50(6):752-7.

3. Rucker NL. $\$ 4$ generics: how low, how broad, and why patient engagement is priceless. J Am Pharm Assoc (2003). 2010;50(6):761-3.

4. Pauly NJ, Brown JD. Prevalence of low-cost generic program use in a nationally representative cohort of privately insured adults. J Manag Care Spec Pharm. 2015;21(12):1162.

5. Low-Cost Generic Program use by medicare beneficiaries: implications for medication exposure misclassification in administrative claims data. J Manag Care Spec Pharm. Forthcoming 2016;22(6).

6. Brown JD, Pauly NJ, Talbert JC. The prevalence and predictors of low-cost generic program use in a nationally representative uninsured population. Pharmacy. 2016;4(1):14.

7. Pauly NJ, Talbert JC, Brown JD. The prevalence and predictors of low-cost generic program use in the pediatric population. Drugs Real World Outcomes. 2015;2(4):411-9.

8. Schneeweiss S, Avorn J. A review of uses of health care utilization databases for epidemiologic research on therapeutics. J Clin Epidemiol. 2005;58(4):323-7.
9. Academy of Managed Care Pharmacy, American Pharmacists A. Medicare star ratings: stakeholder proceedings on community pharmacy and managed care partnerships in quality. J Am Pharm Assoc (2003). 2014;54(3):228-40.

10. Pharmacy Quality Alliance. PQA Performance Measures [homepage on the Internet]. [cited 2016 Apr 5]. Available from: pqaalliance.org/ measures/default.asp.

11. Centers for Medicare \& Medicaid Services. Physician Quality Reporting System [homepage on the Internet]. [cited $2016 \mathrm{Apr}$ 5]. Available from: Www.cms.gov/Medicare/Quality-InitiativesPatient-Assessment-Instruments/PQRS/index. html?redirect=/pqri/

12. Reid RO, Deb P, Howell BL, Shrank WH. Association between Medicare Advantage plan star ratings and enrollment. Jama. 2013;309(3):267-74.

13. Erickson SC, Leslie RS, Patel BV. Is there an association between the high-risk medication star ratings and member experience CMS star ratings measures? J Manag Care Spec Pharm. 2014;20(11):1129-36.

14. Robb MA, Racoosin JA, Sherman RE, et al. The US Food and Drug Administration's Sentinel Initiative: expanding the horizons of medical product safety. Pharmacoepidemiol Drug Saf. 2012;21 Suppl 1:9-11.

15. Blair A, Stewart P, Lubin JH, Forastiere F. Methodological issues regarding confounding and exposure misclassification in epidemiological studies of occupational exposures. Am J Ind Med. 2007; 50(3):199-207.

DOI: $10.5639 /$ gabij.2016.0501.003

Copyright (c) 2016 Pro Pharma Communications International 